\title{
Estimating intrafraction tumor motion during fiducial-based liver stereotactic radiotherapy via an iterative closest point (ICP) algorithm
}

Wu-zhou Li ${ }^{1+}$, Zhi-wen Liang ${ }^{2+}$, Yi Cao ${ }^{1}$, Ting-ting Cao ${ }^{1}$, Hong Quan ${ }^{1}$, Zhi-yong Yang ${ }^{2}$, Qin Li ${ }^{2}$ and Zhi-tao Dai ${ }^{1,3^{*}}$ (I)

\begin{abstract}
Background: Tumor motion may compromise the accuracy of liver stereotactic radiotherapy. In order to carry out a precise planning, estimating liver tumor motion during radiotherapy has received a lot of attention. Previous approach may have difficult to deal with image data corrupted by noise. The iterative closest point (ICP) algorithm is widely used for estimating the rigid registration of three-dimensional point sets when these data were dense or corrupted. In the light of this, our study estimated the three-dimensional (3D) rigid motion of liver tumors during stereotactic liver radiotherapy using reconstructed 3D coordinates of fiducials based on the ICP algorithm.
\end{abstract}

Methods: Four hundred ninety-five pairs of orthogonal kilovoltage (KV) images from the CyberKnife stereo imaging system for 12 patients were used in this study. For each pair of images, the 3D coordinates of fiducial markers inside the liver were calculated via geometric derivations. The 3D coordinates were used to calculate the real-time translational and rotational motion of liver tumors around three axes via an ICP algorithm. The residual error was also investigated both with and without rotational correction.

Results: The translational shifts of liver tumors in left-right (LR), anterior-posterior (AP),and superior-inferior (SI) directions were $2.92 \pm 1.98 \mathrm{~mm}, 5.54 \pm 3.12 \mathrm{~mm}$, and $16.22 \pm 5.86 \mathrm{~mm}$, respectively; the rotational angles in left-right (LR), anteriorposterior (AP), and superior-inferior (SI) directions were $3.95^{\circ} \pm 3.08^{\circ}, 4.93^{\circ} \pm 2.90^{\circ}$, and $4.09^{\circ} \pm 1.99^{\circ}$, respectively. Rotational correction decreased 3D fiducial displacement from $1.19 \pm 0.35 \mathrm{~mm}$ to $0.65 \pm 0.24 \mathrm{~mm}(P<0.001)$.

Conclusions: The maximum translational movement occurred in the SI direction. Rotational correction decreased fiducial displacements and increased tumor tracking accuracy.

Keywords: CyberKnife, Fiducial tracking, SBRT, ICP algorithm, Tumor movement

\section{Introduction}

Traditional radiotherapy can prolong survival for patients with resectable liver cancers [1] but it offers limited efficacy for the treatment of unresectable primary and metastatic liver cancers mainly due to the low whole liver tolerance to radiotherapy [2, 3]. Stereotactic body radiation therapy (SBRT), which is an accurate external beam irradiation method to deliver conformal high doses

\footnotetext{
*Correspondence: daizt_sinap@163.com

${ }^{+}$Wu-zhou Li and Zhi-wen Liang contributed equally to this work.

'School of Physics and Technology, Wuhan University, Wuhan 430022, China

${ }^{3}$ Department of Radiation Oncology, Cancer Hospital Chinese Academy of

Medical Sciences, Shenzhen Center, Shenzhen 518100, China

Full list of author information is available at the end of the article
}

in a few fractions, has been proven to be an effective treatment modality for liver cancers with an elevated rate of local control [3-8].

However, target motion may compromise the accuracy of liver stereotactic radiotherapy. It was reported that liver motions of up to $25 \mathrm{~mm}$ and $55 \mathrm{~mm}$ were observed under normal respiration and deep-breathing, respectively [9-11]. The effect of organ motion on dose has also been investigated [12-14]. According to Velec et al. [14], $70 \%$ patients involved in their study treated with liver stereotactic radiotherapy had accumulated dose deviations relative to the planned static prescription dose > $5 \%$, ranging from -15 to $5 \%$ in tumors and 42 to $8 \%$ in

C The Author(s). 2019 Open Access This article is distributed under the terms of the Creative Commons Attribution 4.0 International License (http://creativecommons.org/licenses/by/4.0/), which permits unrestricted use, distribution, and 
normal tissues. Management of intrafraction motion is crucial to ensure successful liver SBRT so that nearby healthy tissues and critical organs can be spared. Thus, liver tumor translation, rotation and deformation should be considered in both planning and treatment. Many studies have quantified the rigid and non-rigid motions of liver tumors using 4D computed tomography (4DCT) and/or cone beam computed tomography (CBCT) [14-18]. Yet liver tumors are difficult to visualize on $\mathrm{X}$-ray images due to their low contrast against soft tissues around.

An effective solution to this problem is the use of implanted fiducial markers as surrogates of liver tumors [19-21]. Xu et al. [22] proposed a geometric solution to reconstruct the 3D locations of the fiducials and quantified the rigid motion of liver via Least-squares fitting algorithm [23].This is a closed form solution based on singular value decomposition (SVD) of a $3 \times 3$ matrix derived from two point sets. According to Murphy et al. [24], the basic SVD solution is ambiguous in differentiating reflections from rotations especially for the case of only three fiducials (which means point sets are coplanar.) A reflection is a mapping from a Euclidean space to itself that is an isometry with a hyperplane as a set of fixed points. The matrix of a reflection is orthogonal with determinant -1 . In our study, the basic SVD solution yields 175 reflections in 360 trials involving three fiducials. Thus, the basic SVD method must be carefully implemented in the appropriate situation to avoid failures caused by singularities.

According to Euler's rotation theorem, any rotation in three dimensional space can be represented as a combination of a unit vector $\hat{\boldsymbol{e}}$ (called the Euler axis) indicating the direction of an axis of rotation, and an angle $\theta$ describing the magnitude of the rotation about the axis. The quaternions, firstly described by W.R.Hamilton [25] in 1843, give a simple way to encode this axis-angle representation in four numbers. A quaternion representation of rotation can be written as $\hat{\boldsymbol{q}}=q_{i} \boldsymbol{i}+q_{j} \boldsymbol{j}+q_{k} \boldsymbol{k}+q_{r}=\left[\begin{array}{llll}q_{i} & q_{j} & q_{k} & q_{r}\end{array}\right]^{T}$. In terms of the Euler axis $\hat{\boldsymbol{e}}=\left[\begin{array}{lll}e_{x} & e_{y} & e_{z}\end{array}\right]^{T}$ and angle $\theta$, the four components of this quaternion are expressed as follows:

$$
\begin{aligned}
& q_{i}=e_{x} \sin \theta / 2 \\
& q_{j}=e_{y} \sin \theta / 2 \\
& q_{k}=e_{z} \sin \theta / 2 \\
& q_{r}=\cos \theta / 2 .
\end{aligned}
$$

The quaternion-based algorithm doesn't involve with singular value decomposition, which is preferred for our purpose since reflections are not desired. The iterative closest point (ICP) algorithm is a robust and fast algorithm which has been demonstrated useful in estimating real-time prostate motion [26]. In this study, we aim to estimate the intrafractional rigid motion of liver tumor based on real-time KV X-ray images acquired by CyberKnife stereo imaging system via a quaternion-based ICP algorithm.

\section{Material and methods}

This study was approved by the Institutional Review Board at the Tongji Medical College of Huazhong University of Science and Technology. All methods were carried out in accordance with the relevant guidelines and regulations.

\section{Patients and data acquisition}

Twelve patients previously treated for liver cancer with CyberKnife robotic radiotherapy system (Accuray, Inc., Sunnyvale, CA, USA) between 2015 and 2018 were enrolled in this study. Patients and treatment details are summarized in Table 1. Four patients were implanted with four gold fiducials and the remaining eight were implanted with three fiducial markers near the tumor under computed tomography $(\mathrm{CT})$ guidance. Fiducial markers are cylindrical gold seeds with a length of about $3-6 \mathrm{~mm}$ and a diameter of $0.7-1.2 \mathrm{~mm}$. The distance between any two fiducials was greater than $2 \mathrm{~cm}$; and the angle formed by any three fiducials was greater than $15^{\circ}$ to avoid overlapping and collinear effects. These implantation procedures were completed about 1 week before planning CT scan to allow a sufficient time interval for fiducial stabilization. CyberKnife Synchrony fiducial tracking method was used during the whole treatment without breathing control.

\section{Marker segmentation}

The CyberKnife image guidance system consists of two orthogonal X-ray sources fixed on the ceiling and two amorphous silicon panel detectors mounted on both sides of the treatment couch. Figure 1 shows a diagram of the CyberKnife imaging system. The time interval between two adjacent X-ray imaging sessions was $40 \mathrm{~s}$. A total of 495 pairs of KV X-ray images were acquired during the first fraction of the treatment of 12 patients. Each image, a $1024 \times 1024$ matrix, was converted to a binary image containing the number 0.0 (Black) and 1.0 (white). Figure 2a shows a screenshot of a $\mathrm{kV}$ image with three fiducial markers; Fig. 2b shows the image after binarization and mean filtering. The centroid of a fiducial

Table 1 Patient characteristics and main treatment details

\begin{tabular}{ll}
\hline & Mean \pm SD \\
\hline Age $(\mathrm{yr})$ & $58 \pm 12$ \\
Volume $\left(\mathrm{cm}^{3}\right)$ & $5.6 \pm 0.6$ \\
Prescribed dose (Gy) & $44.8 \pm 4.5$ \\
Dose per fraction (Gy) & $9.1 \pm 2.2$ \\
Fraction(n) & $5.1 \pm 1.8$ \\
Duration per fraction (min) & $38.3 \pm 7.4$ \\
\hline
\end{tabular}




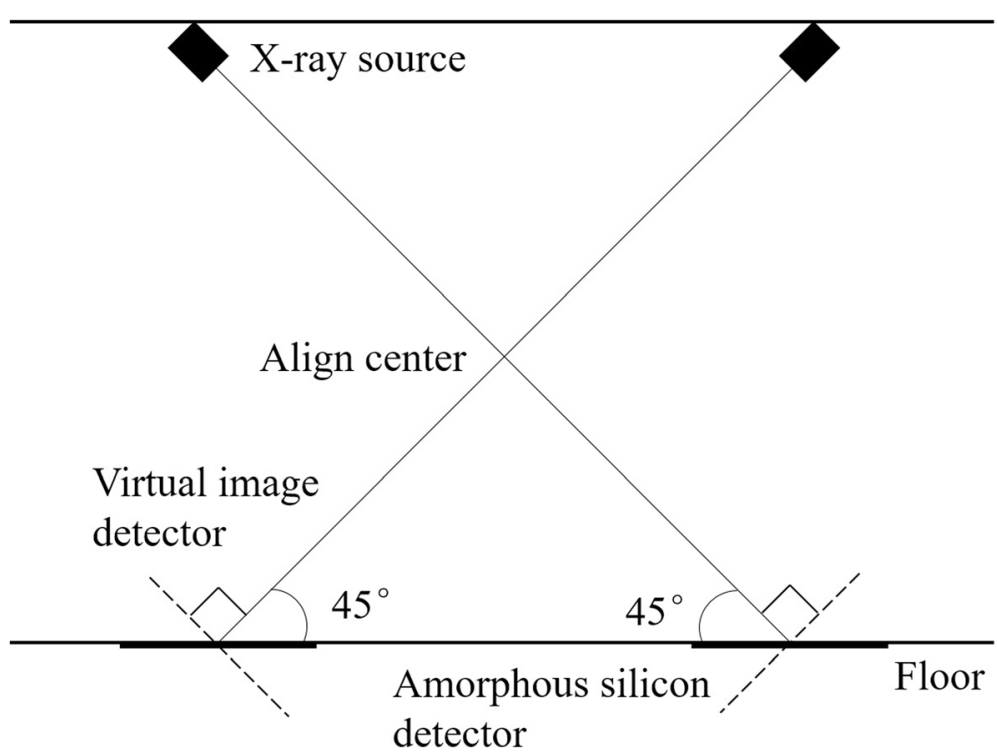

Fig. 1 Diagram of the CyberKnife stereo imaging system

marker was defined at the center point of a 'white blob', and the two-dimensional (2D) position coordinates of the centroid were derived from the binary image.

\section{D fiducial reconstruction}

The fiducial marker is viewed from two orthogonal camera positions, and the 3D coordinates can be reconstructed at the intersection of the back projections towards the source [27]. Parallel rays were assumed for simplicity. A point object (e.g. a fiducial marker) was positioned at the point $\mathrm{M}$ (Fig. 3). Point $P_{A}$ and point $P_{B}$ are projections of point $\mathrm{M}$ on the correspondent image, of which two-dimensional coordinates on the respective image are known. Let the coordinates of the projection point $P_{A}$ be called $\left(u_{a}, v_{a}\right)$, and for point $P_{B}$ be called $\left(u_{b}, v_{b}\right)$. The coordinates of point $\mathrm{M}$ is denoted by $(\alpha$, $\left.\beta^{\prime}, \gamma^{\prime}\right)$ in the image coordinate system $\left(x^{\prime} y^{\prime} z^{\prime}\right)$ and $(\alpha, \beta$, $\gamma$ ) in the patient coordinate system $(x y z)$. The coordinate of both projection points along the SI (Superior-Inferior) direction (z-axis) are theoretically equal. Thus, the 3D coordinates of point $\mathrm{M}$ can be derived as shown in Eq. 2 , after combining geometrical information.

$$
\begin{aligned}
\left(\begin{array}{l}
x \\
y \\
z
\end{array}\right)= & \left(\begin{array}{ccc}
\cos \theta & -\sin \theta & 0 \\
\sin \theta & \cos \theta & 0 \\
0 & 0 & 1
\end{array}\right) \\
& \times\left(\begin{array}{c}
u_{a} \\
u_{b} \\
\left(v_{a}+v_{b}\right) / 2
\end{array}\right)
\end{aligned}
$$

The reconstruction algorithm was validated using the stereotactic dose verification phantom (SDVP; Standard Imaging, Inc., Middleton, WI, USA), which was illustrated in Additional file 1. The results show that the mean difference between the reconstructed 3D fiducial coordinates and those recorded in the CyberKnife log file is $0.72 \mathrm{~mm}$.
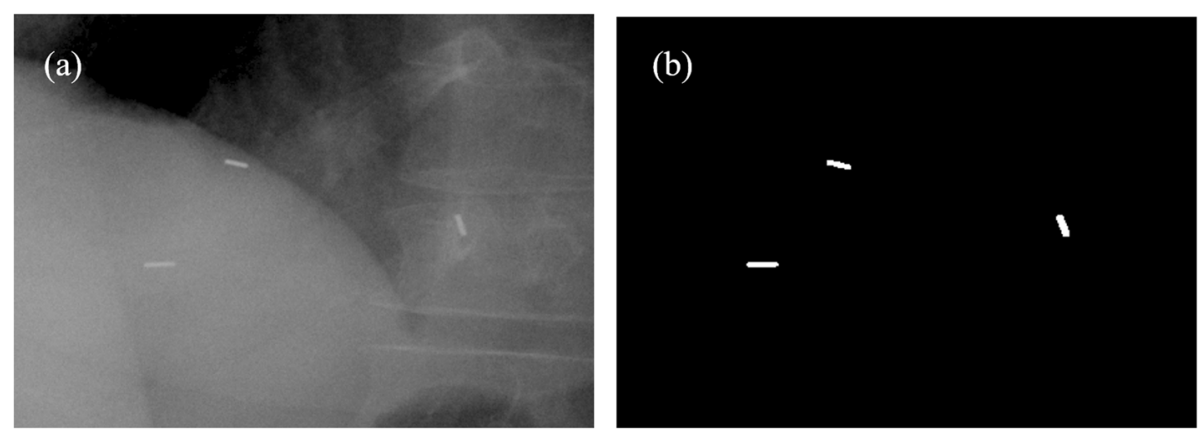

Fig. 2 a Two-dimensional kV image of three radio opaque fiducial markers. b Bitmap obtained after binarization and mean filtering 


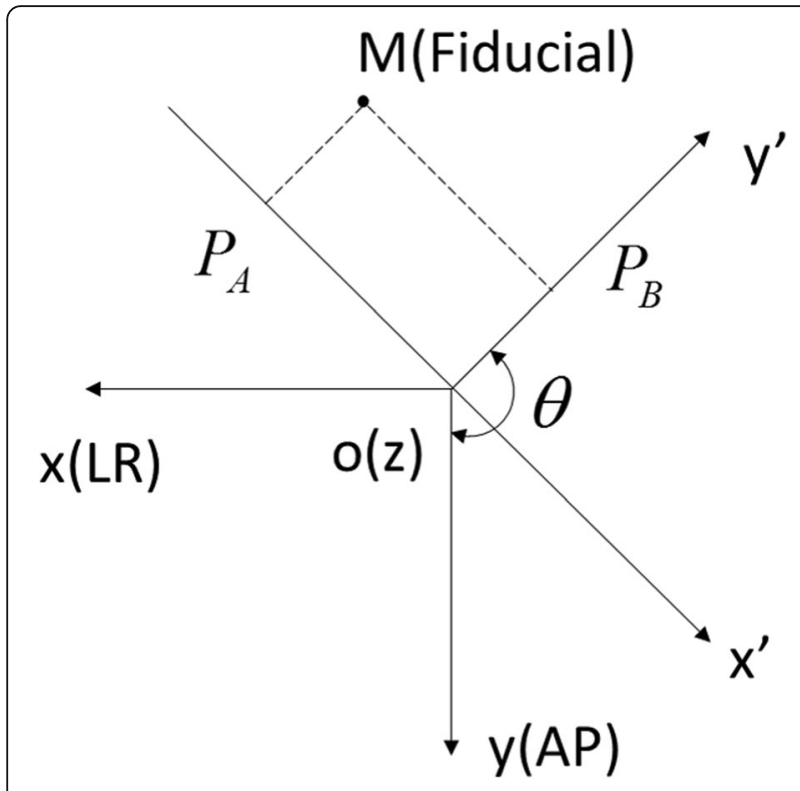

Fig. 3 Diagram showing projection and coordinate system rotation. The fiducial marker is at $\mathrm{M}_{;} P_{A}$ and $P_{B}$ are the two projection points

\section{Estimation of translational and rotational motions}

Assuming two sets of data points for fiducial markers, the set of target data points (denoted as $Y=\left\{y_{1}, y_{2}, \ldots y_{n}\right\}$ ) was translated and rotated to the set of reference data points (denoted as $\left.\mathrm{X}=\left\{x_{1}, x_{2}, \ldots x_{n}\right\}\right)$. Due to liver tumor deformation and fiducial marker migration, it is not possible to find a transformation that perfectly maps the two sets of fiducial markers. The aim of the ICP algorithm was to find the rotation matrix $R$ and translation vector $T$ that minimizes the following objective function:

$$
\sum^{2}=\frac{1}{n} \sum_{i=1}^{n}\left\|x_{i}-\left(R y_{i}+T\right)\right\|^{2}
$$

The flow chart of the ICP algorithm is shown in Fig. 4.

Step 1: the ICP algorithm is based on the nearest neighbor decision rule [28] to match up the corresponding points.

Step 2 and 3: computed the rotation matrix $R$ and translation vector $T$ that minimizes the mean square error of the estimated corresponding pairs.

Step 4: the threshold was set to $0.001 \mathrm{~mm}$. Typically, it takes no more than 2 or 3 iterations before achieving convergence.

The derivation of the quaternion-based ICP algorithm used in our study is described as follows: Assuming the rotation matrix $R$ is denoted by $R=q=\left[\begin{array}{llll}q_{0} & q_{1} & q_{2} & q_{3}\end{array}\right]^{T}$.

The centroids of point set $X$ and point set $Y$ are given by

$$
\left\{\begin{array}{l}
\mu_{x}=\frac{1}{n} \sum_{i=1}^{n} x_{i} \\
\mu_{y}=\frac{1}{n} \sum_{i=1}^{n} y_{i}
\end{array}\right.
$$

and the cross-covariance matrix of sets $Y$ and $X$ can be written as follows:

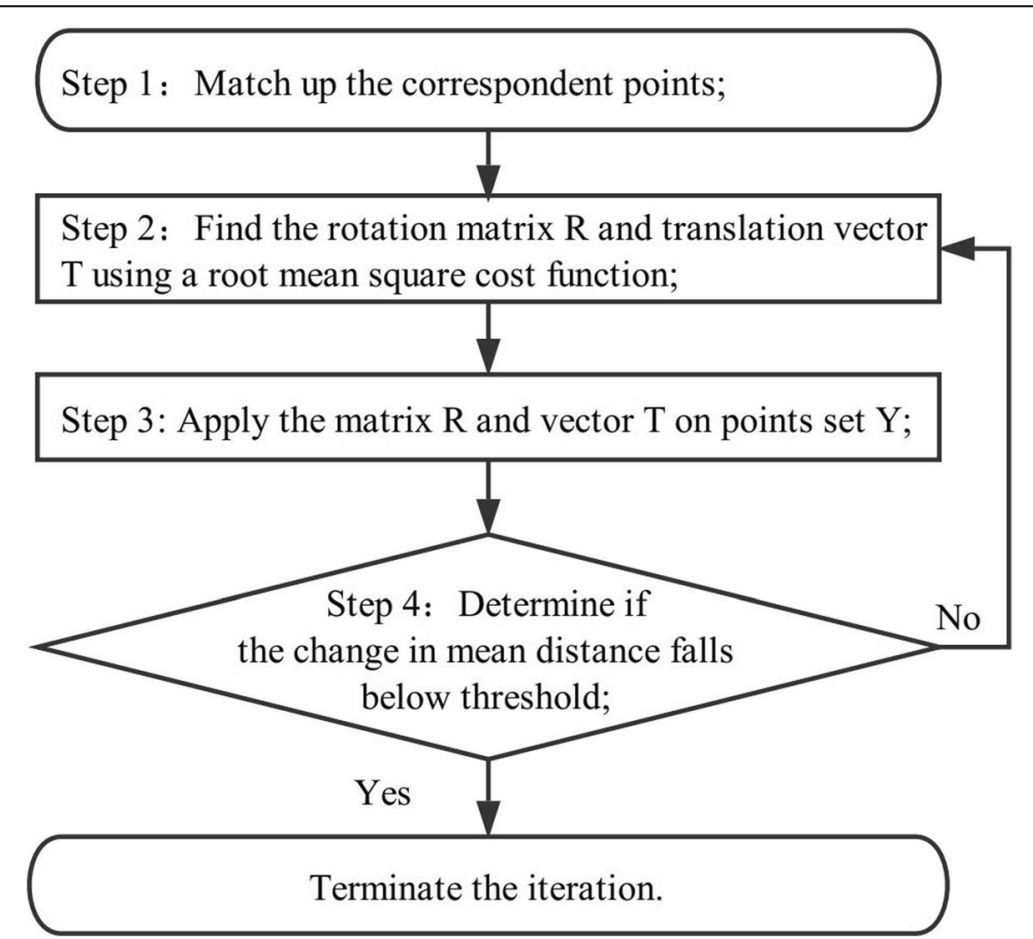

Fig. 4 Flow chart of the ICP algorithm implemented in 3D fiducial registration 
Table 2 The mean and standard deviation (SD) of translational and rotational motion ranges of liver tumors in each direction

\begin{tabular}{llllllll}
\hline & $\Delta x(\mathrm{~mm})$ & $\Delta y(\mathrm{~mm})$ & $\Delta z(\mathrm{~mm})$ & $\Delta \mathrm{d}(\mathrm{mm})$ & $\Delta \theta_{x}\left({ }^{\circ}\right)$ & $\Delta \theta_{\mathrm{y}}\left({ }^{\circ}\right)$ & $\Delta \theta_{z}\left({ }^{\circ}\right)$ \\
\hline Mean & 2.92 & 5.54 & 16.22 & 11.89 & 3.95 & 4.93 & 4.09 \\
SD & 1.98 & 3.12 & 5.86 & 5.11 & 3.08 & 2.90 & 1.99 \\
\hline
\end{tabular}

$$
\begin{aligned}
\sum_{y x} & =\frac{1}{n} \sum_{i=1}^{n}\left[\left(y_{i}-\mu_{y}\right)\left(x_{i}-\mu_{x}\right)^{T}\right] \\
& =\frac{1}{n} \sum_{i=1}^{n} y_{i} x_{i}{ }^{T}-\mu_{y} \mu_{x}{ }^{T}
\end{aligned}
$$

Construct matrix A:

$$
A_{i j}=\left(\sum_{y x}-\sum_{y x}^{T}\right)_{i j}
$$

Matrix $\mathrm{A}$ is used to construct the column vector $\Delta$ :

$$
\Delta=\left[\begin{array}{lll}
A_{23} & A_{31} & A_{12}
\end{array}\right]^{T}
$$

Vector $\Delta$ is applied to yield a symmetric matrix Q:

$$
Q=\left[\begin{array}{cc}
\operatorname{tr}\left(\sum_{y x}\right) & \Delta^{T} \\
\Delta & \sum_{y x}+\sum_{y x}^{T}-t r\left(\sum_{y x}\right) I_{3}
\end{array}\right]
$$

where $\operatorname{tr}(\bullet)$ denotes the trace of a matrix, and $I_{3}$ is a $3 \times$ 3 identity matrix.

According to the studies of Besl et al. [29] and Horn et al. [30], the unit eigenvector $q=\left[\begin{array}{llll}q_{0} & q_{1} & q_{2} & q_{3}\end{array}\right]^{T}$ corresponding to the maximum eigenvalue of the matrix $Q$ is regarded as the quaternion that minimizes the objective function (3). According to $q$, the rotation matrix $R$ can be written as:

$$
R=\left[\begin{array}{ccc}
q_{0}^{2}+q_{1}^{2}-q_{2}^{2}-q_{3}^{2} & 2\left(q_{1} q_{2}-q_{0} q_{3}\right) & 2\left(q_{1} q_{3}+q_{0} q_{2}\right) \\
2\left(q_{1} q_{2}+q_{0} q_{3}\right) & q_{0}^{2}+q_{2}^{2}-q_{1}^{2}-q_{3}^{2} & 2\left(q_{2} q_{3}-q_{0} q_{1}\right) \\
2\left(q_{1} q_{3}-q_{0} q_{2}\right) & 2\left(q_{2} q_{3}+q_{0} q_{1}\right) & q_{0}^{2}+q_{3}^{2}-q_{1}^{2}-q_{2}^{2}
\end{array}\right]
$$

After $\mathrm{R}$ was solved, the translation vector $T$ could be derived:

$$
T=\mu_{x}-R \mu_{y}
$$

\section{Statistical analyses}

The fiducials in a pair of orthogonal images near the beginning of each treatment were used as reference points set, and the registration residual errors with translational
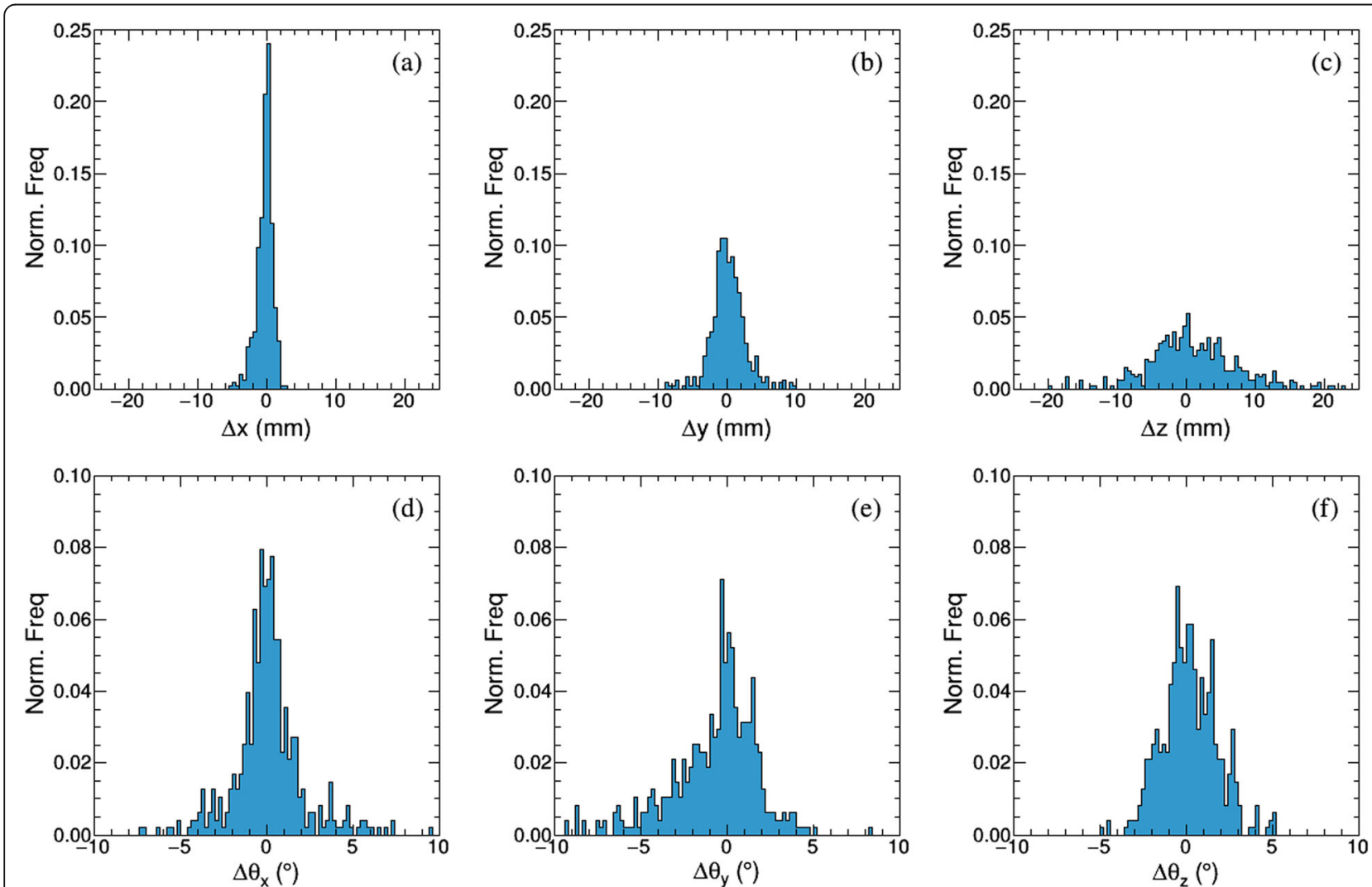

Fig. 5 Normalized histogram of translational movements in the (a) Left-Right LR $(\Delta \mathrm{x})$, (b) Anterior-Posterior AP $(\Delta \mathrm{y})$, (c) Superior-Inferior SI $(\Delta \mathrm{z})$ directions respectively. Normalized histogram of rotational movements in the (d) Left-Right $L R\left(\Delta \theta_{x}\right)$, (e) Anterior-Posterior AP $\left(\Delta \theta_{y}\right)$, (f) SuperiorInferior SI $\left(\Delta \theta_{z}\right)$ directions, respectively 

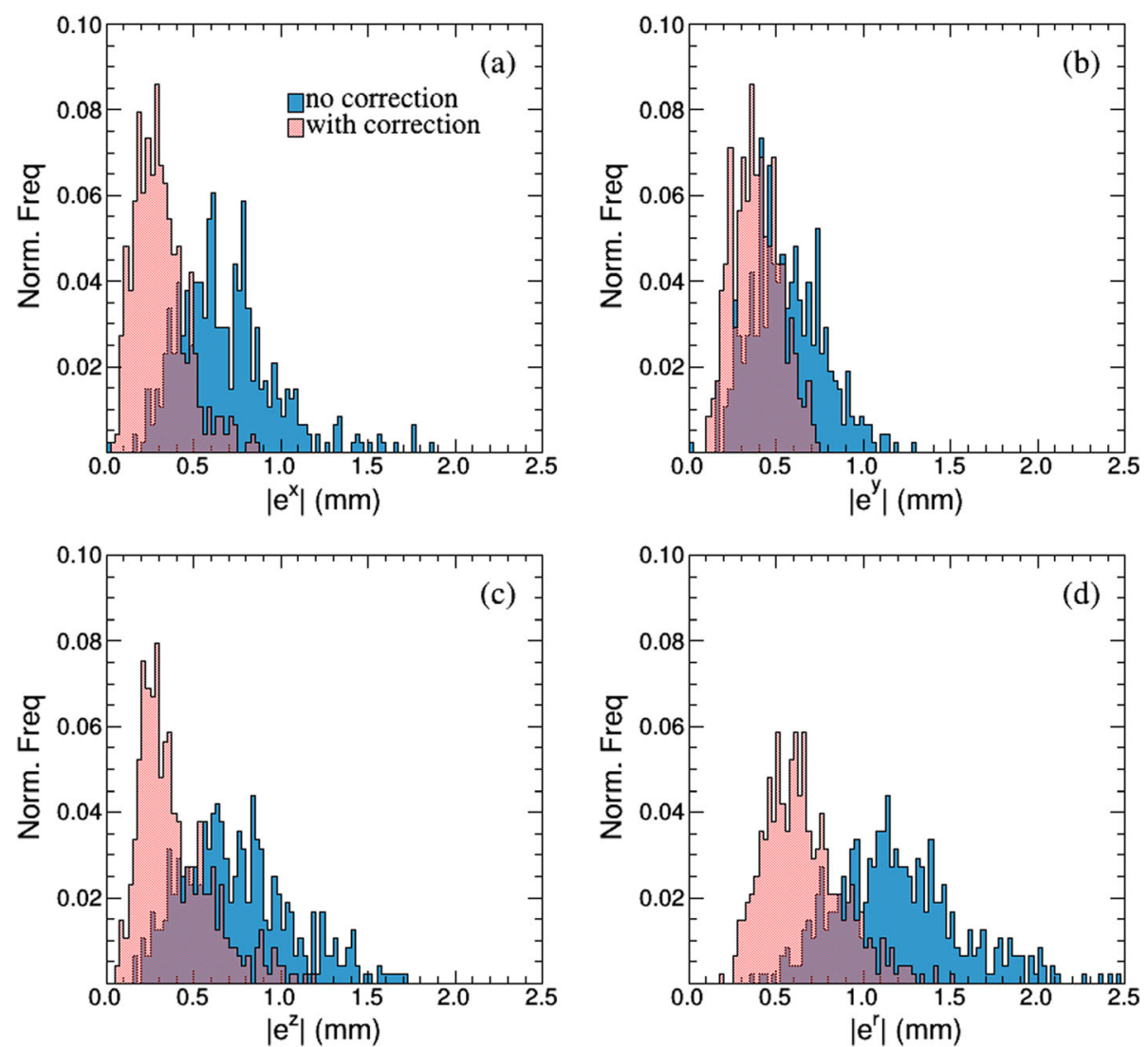

Fig. 6 Fiducial registration residual errors in the (a) Left-Right LR $\left(\left|e^{x}\right|\right)$, (b) Anterior-Posterior AP $\left(\left|e^{y}\right|\right)$, (c) Superior-Inferior SI (|ez $)$ directions, respectively. (d) Residual errors in 3D space $\left(\left|e^{r}\right|\right)$. The blue area indicates with translational corrections only; the red area indicates with rigid corrections

corrections only and with rigid corrections are also recorded. Statistical analysis was performed using paired sample t-test analysis with SPSS Statistics 20 (IBM, Armonk, USA) software. The null hypothesis is that the true difference between registration errors with translational corrections only and with rigid corrections is zero. $P$ value $<0.001$ was considered statistically significant at 95\% confidence level.

\section{Results}

A total of 495 pairs of $\mathrm{kV} \mathrm{X-ray} \mathrm{images} \mathrm{from} 12$ patients were analyzed in this study. The translational and rotational motion ranges are summarized in Table 2. Translation and rotation are measured as the mean of the maximum range of motion for each case. For all patients, the translational motion ranges in left-right (LR), anterior-posterior (AP), and superior-inferior (SI) directions were $2.92 \pm 1.98 \mathrm{~mm}(\Delta \mathrm{x}), 5.53 \pm 3.12 \mathrm{~mm}(\Delta \mathrm{y})$, and $16.22 \pm 5.86 \mathrm{~mm}(\Delta \mathrm{z})$, respectively. Translational motion range in $3 \mathrm{D}$ space $(\Delta \mathrm{d})$ can be computed as:

$$
\Delta \mathrm{d}=\sqrt{\Delta \mathrm{x}^{2}+\Delta \mathrm{y}^{2}+\Delta \mathrm{z}^{2}}
$$

The translational motion range in $3 \mathrm{D}$ space was $11.89 \pm 5.11 \mathrm{~mm}(\Delta \mathrm{d})$.

The rotational angles in LR, AP, and SI directions were $3.95^{\circ} \pm 3.08^{\circ}\left(\Delta \theta_{x}\right), 4.93^{\circ} \pm 2.90^{\circ}\left(\Delta \theta_{y}\right)$, and $4.09^{\circ} \pm$

Table 3 Residual error with and without (w/o) rotational corrections. Statistical analysis was performed using paired sample t-test analysis

\begin{tabular}{llll}
\hline & $\begin{array}{l}\text { Error w/o rotational correction } \\
\text { Mean } \pm \text { SD }\end{array}$ & $\begin{array}{l}\text { Error with rotational correction } \\
\text { Mean } \pm \text { SD }\end{array}$ & $\begin{array}{c}p^{-} \\
\text {value }\end{array}$ \\
\hline$\left|\mathrm{e}^{\mathrm{x}}\right|(\mathrm{mm})$ & $0.68 \pm 0.26$ & $0.31 \pm 0.15$ & $<0.001$ \\
$\left|\mathrm{e}^{\mathrm{y}}\right|(\mathrm{mm})$ & $0.39 \pm 0.13$ & $<0.001$ \\
$\left|\mathrm{e}^{\mathrm{z}}\right|(\mathrm{mm})$ & $0.57 \pm 0.21$ & $0.39 \pm 0.21$ & $<0.001$ \\
$\left|\mathrm{e}^{\mathrm{d}}\right|(\mathrm{mm})$ & $0.74 \pm 0.31$ & $0.65 \pm 0.23$ & $<0.001$ \\
\hline
\end{tabular}


$1.99^{\circ}\left(\Delta \theta_{\mathrm{z}}\right)$, respectively. Figure 5 displays a normalized frequency histogram of intrafractional translational shift and rotational angles of all patients. Large rotation angles exceeding $8^{\circ}$ were only observed in two cases. Figure 6 shows the registration residual error between the reference points and the transformed target points with rigid corrections (Fig. 6, red) and with translational corrections only (Fig. 6, blue). The mean \pm SD of residual errors with and without rotational correction in each direction are displayed in the Table 3 . The difference in each direction was statistically significant with and without rotational correction $(P<0.001)$. Rotational corrections decreased 3D residual error from $1.19 \pm 0.35 \mathrm{~mm}$ to $0.68 \pm 0.24 \mathrm{~mm}$.

\section{Discussion}

In this study, the intrafraction translations and rotations of liver tumor were estimated via the ICP algorithm during CyberKnife-based stereotactic radiotherapy. The maximum translational motion occurred in the SI direction due to breathing motion, and the smallest translation was observed along the LR direction, which agrees with previous studies $[15,18,20,21]$. Xu et al. [22] obtained $\mathrm{kV}$ images from CyberKnife imaging system and reported that the means \pm SD of the absolute value of intrafraction translations in liver SBRT was $2.1 \pm 2.3 \mathrm{~mm}$ (LR), $2.9 \pm 2.8 \mathrm{~mm}$ (AP), and $6.4 \pm 5.5 \mathrm{~mm}$ (SI). Higher values were obtained in this study, since the maximum motion range for each patient was used to calculate the mean and standard deviation of both translation and rotation. In addition to translational movements, large rotational motion angles were observed. This suggests that the rigid motion of liver tumor should be paid special attention to for some patients. The residual error with rotational correction was $0.65 \pm 0.23 \mathrm{~mm}$, probably due to liver tumor deformation during treatment.

Several ways can be utilized to compute the optimal rigid transformation of two geometric data sets. Matching up the correspondent point one-to-one between the reference image and the test image is necessary to solve this problem. Distance between fiducials should exceed $20 \mathrm{~mm}$ to avoid overlapping or mismatching [24]. It is not difficult to associate the target point with the corresponding point in reference image in our case. Once the correspondence is known, the orthogonal transformation that minimizes the residual error in points set registration can be found with both of the closed form and iterative methods. This indicates that true least-squares minimum exists in the solution and the convergence of the iterative algorithm is guaranteed. The closest iterative point (ICP) algorithm is commonly used in medical image registration [31,32], and it was found to be more general and robust when dealing with corrupted data which is more common in realistic scenarios.
The algorithm for fiducial segmentation in this study might introduce minor errors. Firstly, the resolution of $\mathrm{kV}$ images obtained by CyberKnife imaging system was $1024 \times 1024$. Thus, the centroid of the 'white blobs' calculated by the coordinates of the pixel block may deviate from the actual centroid of the gold fiducial markers. Secondly, the process of binarization could also introduce minor errors to the calculation of the fiducial centroids. Automatic solutions to segment radiopaque fiducial markers from CBCT scans have been proposed previously [27, 33, 34]. Mao et al. [33] presented a pattern matching algorithm using matched filters and template matching for detecting fiducials specifically designed to work with both $\mathrm{kV}$ and MV image data. However, this algorithm might be less applicable in this study because the projections of fiducials change with projection angle caused by liver deformation. Other solutions using CBCT projections with large angular separation and good marker contrast on a uniform background might also be not suitable in this study. Considering that the image data used in this study contained considerable noise caused by the bone structure, false signals are almost inevitable with these methods. Fiducial trajectory estimation is important in tumor motion management such as tumor tracking. A robust and reliable automatic segmentation method used in CyberKnife-based kV X-ray images needs to be developed.

\section{Conclusion}

Tumor motion management is necessary to improve the accuracy of SBRT. The rotational and translational motions of liver tumors during SBRT were estimated based on the CyberKnife system via the ICP algorithm. The residual error after registration decreased significantly with rotational correction. The results of this study can be used in motion management and planning target volume (PTV) margin determination for both liver SBRT and conventional radiotherapy.

\section{Supplementary information}

Supplementary information accompanies this paper at https://doi.org/10. 1186/s13014-019-1401-2.

Additional file 1. The detailed information about SDVP.

\begin{abstract}
Abbreviations
3D: Three-dimensional; 4DCT: 4D computed tomography; AP: Anteriorposterior; CBCT: Cone beam computed tomography; CT: Computed tomography; ICP: Iterative closest point; KV: Kilovoltage; LR: Left-right; MV: Megavoltage; PTV: Planning target volume; SBRT: Stereotactic body radiation therapy; SDVP: Stereotactic dose verification phantom; SI: Superiorinferior; SVD: Singular value decomposition
\end{abstract}

Acknowledgements

No Acknowledgement. 


\section{Authors' contributions}

Conception and design: Z-TD, Z-WL Acquisition of data: W-ZL, Z-WL Analysis of data: W-ZL, YC Writing, review and/or revision of the manuscript: W-ZL, ZTD, Z-YY, QL, T-TC All authors reviewed the manuscript. All authors read and approved the final manuscript.

\section{Funding}

This work is supported by the National Natural Science Foundation of China (Grant No.81803047) the China Postdoctoral Science Foundation (Grant No.2018 M640725)

\section{Availability of data and materials}

All data included in this study are available upon request by contact with the corresponding author.

\section{Ethics approval and consent to participate}

This study was approved by the Institutional Review Board at the Tongji Medical College of Huazhong University of Science and Technology. All methods were carried out in accordance with the relevant guidelines and regulations.

\section{Consent for publication}

Not applicable.

\section{Competing interests}

The authors declare that they have no competing interests.

\section{Author details}

'School of Physics and Technology, Wuhan University, Wuhan 430022, China. ${ }^{2}$ Cancer Center, Union Hospital, Tongji Medical College, Huazhong University of Science and Technology, Wuhan 430022, China. ${ }^{3}$ Department of Radiation Oncology, Cancer Hospital Chinese Academy of Medical Sciences, Shenzhen Center, Shenzhen 518100, China.

\section{Received: 30 June 2019 Accepted: 16 October 2019}

\section{Published online: 29 October 2019}

\section{References}

1. Wei $X$, Jiang $Y$, Zhang $X$, et al. Neoadjuvant three-dimensional conformal radiotherapy for Resectable hepatocellular carcinoma with portal vein tumor Thrombus: a randomized, open-label, Multicenter Controlled Study. J Clin Oncol. 2019;37(24):2141.

2. Dawson LA, Ten Haken RK, Lawrence TS. Partial irradiation of the liver. Semin Radiat Oncol. 2001;11(3):240-6.

3. Zhang SY, Zhu GY, Li G, Zhang YB, Geng JH. Application of stereotactic body radiation therapy to cancer liver metastasis. Cancer Lett. 2016;379(2): 225-9.

4. Wulf J, Guckenberger M, Haedinger U, Oppitz U, Mueller G, Baier K, Flentje M. Stereotactic radiotherapy of primary liver cancer and hepatic metastases. Acta Oncol. 2006:45(7):838-47.

5. Lee MT, Kim JJ, Dinniwell R, Brierley J, Lockwood G, Wong R, Cummings B, Ringash J, Tse RV, Knox JJ, Dawson LA. Phase I study of individualized stereotactic body radiotherapy of liver metastases. J Clin Oncol. 2009;27(10):1585-91.

6. Aitken $\mathrm{KL}$, Hawkins M. A: stereotactic body radiotherapy for liver metastases. Clin Oncol. 2015;27(5):307-15

7. Fundowicz M, Adamczyk M, Kołodziej-Dybaś A. Stereotactic body radiation therapy for liver metastasis - the linac-based greater Poland cancer Centre practice. Rep Pract Oncol Radiother. 2017;22(2):158-62.

8. Koay EJ, Owen D, Das P. Radiation-induced liver disease and modern radiotherapy. Semin Radiat Oncol. 2018;28(4):321-31.

9. Suramo I, Päivänsalo M, Myllylä V. Cranio-caudal movements of the liver, pancreas and kidneys in respiration. Acta Radiol Diagn (Stockh). 1984;25(2):129-31.

10. Langen KM, Jones DTL. Organ motion and its management. Int J Radiat Oncol Biol Phys. 2001;50(1):265-78.

11. Tanguturi SK, Wo JY, Zhu AX, Dawson LA, Hong TS. Radiation therapy for liver tumors: ready for inclusion in guidelines? Oncologist. 2014;9(8):868-79.

12. Rosu M, Dawson LA, Balter JM, McShan DL, Lawrence TS, Ten Haken RK. Alterations in normal liver doses due to organ motion. Int J Radiat Oncol Biol Phys. 2003;57(5):1472-9.

13. Velec M, Moseley JL, Eccles CL, Craig T, Sharpe MB, Dawson LA, Brock KK. Effect of breathing motion on radiotherapy dose accumulation in the abdomen using deformable registration. Int J Radiat Oncol Biol Phys. 2011; 80(1):265-72.

14. Velec M, Moseley JL, Craig T, Dawson LA, Brock KK. Accumulated dose in liver stereotactic body radiotherapy: positioning, breathing, and deformation effects. Int J Radiat Oncol Biol Phys. 2012;83(4):1132-40.

15. Case RB, Sonke JJ, Moseley DJ, Kim J, Brock KK, Dawson LA. Inter- and intrafraction variability in liver position in non-breath-hold stereotactic body radiotherapy. Int J Radiat Oncol Biol Phys. 2009;75(1):302-8.

16. Eccles $\mathrm{CL}$, Dawson LA, Moseley $\mathrm{J}$, Brock KK. Interfraction liver shape variability and impact on GTV position during liver stereotactic radiotherapy using abdominal compression. Int J Radiat Oncol Biol Phys. 2011;80(3):938-46.

17. Cao M, Lasley FD, Das IJ, Desrosiers CM, Slessinger ED, Cardenes HR. Evaluation of rotational errors in treatment setup of stereotactic body radiation therapy of liver cancer. Int J Radiat Oncol Biol Phys. 2012;84(3): e435-40.

18. Bertholet J, Worm ES, Fledelius W, Høyer M, Poulsen PR. Time-resolved intrafraction target translations and rotations during stereotactic liver radiation therapy: implications for marker-based localization accuracy. Int J Radiat Oncol Biol Phys. 2016;95(2):802-9.

19. Saw $\mathrm{CB}$, Chen $\mathrm{H}$, Wagner $\mathrm{H}$ Jr. Implementation of fiducial-based image registration in the Cyberknife robotic system. Med Dosim. 2008;33(2):156-60

20. Park JC, Park SH, Kim JH, Yoon SM, Song SY, Liu Z, Song B, Kauweloa K, Webster MJ, Sandhu A, Mell LK, Jiang SB, Mundt AJ, Song WY. Liver motion during cone beam computed tomography guided stereotactic body radiation therapy. Med Phys. 2012;39(10):6431-42.

21. Shimohigashi Y, Toya R, Saito T, Ikeda O, Maruyama M, Yonemura K, Nakaguchi $Y$, Kai Y, Yamashita Y, Oya N, Araki F. Tumor motion changes in stereotactic body radiotherapy for liver tumors: an evaluation based on four-dimensional cone-beam computed tomography and fiducial markers. Radiat Oncol. 2017;12(1):61.

22. Xu Q, Hanna G, Grimm J, Kubicek G, Pahlajani N, Asbell S, Fan J, Chen Y, LaCouture T. Quantifying rigid and nonrigid motion of liver tumors during stereotactic body radiation therapy. Int J Radiat Oncol Biol Phys. 2014;90(1): 94-101.

23. Arun KS, Huang TS, Blostein SD. Least-squares fitting of two 3-d point sets. IEEE Trans Pattern Anal Mach Intell. 1987:9(5):698-700.

24. Murphy MJ. Fiducial-based targeting accuracy for external-beam radiotherapy. Med Phys. 2002;29(3):334-44.

25. Professor Sir William Rowan Hamilton LL.D. V.P.R.I.A. F.R.A.S. XXXVI. On quaternions; or on a new system of imaginaries in algebra. Philosophical Magazine Series 3, 31(207):214-219.

26. Tehrani JN, O'Brien RT, Poulsen PR, Keall P. Real-time estimation of prostate tumor rotation and translation with a kv imaging system based on an iterative closest point algorithm. Phys Med Biol. 2013;58(23):8517-33.

27. Park SJ, Ionascu D, Hacker F, Mamon H, Berbeco R. Automatic marker detection and 3D position reconstruction using cine EPID images for SBRT verification. Med Phys. 2009;36(10):4536-46.

28. Cover T, Hart P. Nearest neighbor pattern classification. IEEE Trans Inf Theory. 1967;13(1):21-7.

29. Besl P, McKay ND. A method for registration of 3-D shapes. IEEE Trans Pattern Anal Mach Intell. 1992;14(2):239-56.

30. Horn BKP. Closed-form solution of absolute orientation using unit quaternions. J Opt Soc Am A. 1987;4(2):629-42.

31. Almhdie $A$, Léger $C$, Deriche $M$, Lédée $R$. $3 D$ registration using a new implementation of the ICP algorithm based on a comprehensive lookup matrix: application to medical imaging. Pattern Recogn Lett. 2007;28(12): 1523-33.

32. Pan M-s, Tang J-t, Rong Q-s, Zhang F. Medical image registration using modified iterative closest points. Int J Numer Method Biomed Eng. 2011; 27(8):1150-66

33. Mao W, Wiersma RD, Xing L. Fast internal marker tracking algorithm for onboard mv and kv imaging systems. Med Phys. 2008;35(5):1942-9.

34. Poulsen PR, Fledelius W, Keall PJ, Weiss E, Lu J, Brackbill E, Hugo GD. A method for robust segmentation of arbitrarily shaped radiopaque structures in cone-beam CT projections. Med Phys. 2011;38(4):2151-6.

\section{Publisher's Note}

Springer Nature remains neutral with regard to jurisdictional claims in published maps and institutional affiliations. 